A Discrete-Time Solution to Wave Propagation in

Time-Varying Media

\author{
Shyh-Kang Jeng \\ Department of Electrical Engineering and \\ Graduate Institute of Communication Engineering, \\ National Taiwan University, Taipei, Taiwan \\ email: skjeng@ew.ee.ntu.edu.tw
}

\begin{abstract}
A novel approximate solution to wave propagation in time-varying media is proposed. This closed-form solution is derived using the discrete-time approach. It is written down directly from the corresponding frequency domain solution in time-invariant media with simple duality nules. This approach can be applied to develop other analytical as well as numerical solution techniques for wave problems in time-varying media.
\end{abstract}

\title{
Introduction
}

Time-varying media at least can be found in ferroelectric material, magnetoelastic material, general space-time periodic media, and plasma suddenly created by solar flares, strong laser pulse, or by nuclear explosion. The electromagnetic wave propagation in such media is often regarded as a difficult problem. The solution techniques developed before, as far as the author knows, include analytical solutions to differential equations, the WKB ray approximation, the integral equation approach, and the finite-difference time-domain method. Typical related references are [1][2].

This paper will propose a new solution based on the discrete-time approach, which is an extension of the discrete-time electromagnetic theory [3] and will be introduced in the next section. By this approach, a closed form approximate solution for homogeneous gradually-time-varying media is obtained in a straightforward way. This approach can be developed further to derive new analytical as well as numerical methods for solving wave problems 
in time-varying media.

\section{Discrete-Time Approach}

Supposed that a continuous signal $s(t)$ is sampled as a sequence $s_{n}$ at $n \Delta t,-\infty<n<\infty$. Its derivative at $n \Delta t$ can be approximated by $s^{\prime}(n \Delta t) \approx D s_{n}=\frac{1}{\Delta t} \sum_{i=k}^{m} d_{i} s_{n-i}$, where $k, m$, and $d_{i}$ 's depend on the method of approximation used (for example, Lagrange polynomial interpolation, staircase-approximation time-domain (SATD) [3][4], etc.) By frequency analysis and numerical experiments, it is found that a set of parameters corresponding to the four-point Lagrange polynomial interpolation, i.e., $k=0$, $m=3, d_{0}=11 / 6, d_{1}=-18 / 6, d_{2}=9 / 6, d_{3}=-2 / 6$ often leads to satisfactory results, if the high frequency components of the signal is not very significant. The discrete-time approximation of the derivative can also be written in a matrix form:

$$
\left[\begin{array}{c}
\vdots \\
D s_{0} \\
D s_{1} \\
D s_{2} \\
\vdots
\end{array}\right]=\frac{1}{\Delta t}\left[\begin{array}{ccccc}
\ddots & \vdots & \vdots & \vdots & \cdot \\
\cdots & d_{0} & d_{-1} & d_{-2} & \cdots \\
\cdots & d_{1} & d_{0} & d_{-1} & \cdots \\
\cdots & d_{2} & d_{1} & d_{0} & \cdots \\
\cdot & \vdots & \vdots & \vdots & \ddots
\end{array}\right]\left[\begin{array}{c}
\vdots \\
s_{0} \\
s_{1} \\
s_{2} \\
\vdots
\end{array}\right],
$$

or $[D s]=\frac{1}{\Delta t}[D[s]$, where the matrix $[D]$ is an infinite Toeplitz matrix.

Let the electromagnetic field quantities be sampled at $n \Delta t,-\infty<n<\infty$. Using the discrete-time approach given above, the Maxwell equations can be represented as

$$
\begin{aligned}
& \nabla \times[\bar{E}(\bar{r})]=-\frac{1}{\Delta t}[D[\bar{B}(\bar{r})]-[\bar{M}(\bar{r})] \\
& \nabla \times[\bar{H}(\bar{r})]=\frac{1}{\Delta t}[D[\bar{D}(\bar{r})]+[\bar{J}(\bar{r})] \\
& \nabla \cdot[\bar{D}(\bar{r})]=\left[\rho_{e}(\bar{r})\right] \\
& \nabla \cdot[\bar{B}(\bar{r})]=\left[\rho_{m}(\bar{r})\right]
\end{aligned}
$$

The time-varying constitution relationship 


$$
\begin{aligned}
& \overline{\mathrm{D}}(\bar{r}, t)=\varepsilon_{0} \varepsilon_{\infty}(\bar{r}, t) \overline{\mathrm{E}}(\bar{r}, t)+\varepsilon_{0} \int_{-\infty} \chi_{e}\left(\bar{r}, t, t^{\prime}\right) \overline{\mathrm{E}}\left(\bar{r}, t^{\prime}\right) d t^{\prime} \\
& \overline{\mathrm{B}}(\bar{r}, t)=\mu_{0} \mu_{\infty}(\bar{r}, t) \overline{\mathrm{H}}(\bar{r}, t)+\mu_{0} \int_{-\infty}^{1} \chi_{m}\left(\bar{r}, t, t^{\prime}\right) \overline{\mathrm{H}}\left(\bar{r}, t^{\prime}\right) d t^{\prime} \\
& \overline{\mathrm{J}}_{c}(\bar{r}, t)=\sigma_{e}(\bar{r}, t) \overline{\mathrm{E}}(\bar{r}, t) \\
& \overline{\mathrm{M}}_{c}(\bar{r}, t)=\sigma_{m}(\bar{r}, t) \overline{\mathrm{H}}(\bar{r}, t)
\end{aligned}
$$

can be also expressed in a matrix form

$$
\begin{aligned}
& \left.[\bar{D}]=\varepsilon_{0}[\varepsilon] \bar{E}\right] \\
& \left.[\bar{B}]=\mu_{0}[\mu] \bar{H}\right], \\
& {\left[\bar{J}_{c}\right]=\left[\sigma_{e} \backslash \bar{E}\right],} \\
& {\left[\bar{M}_{c}\right]=\left[\sigma_{m}[\bar{H}]\right.}
\end{aligned}
$$

where the matrix elements $\varepsilon_{i j}=\varepsilon_{\infty ; i} \delta_{i j}+w_{i, j} \chi_{e ; i, j}, \mu_{i j}=\mu_{\infty ; i} \delta_{i j}+w_{i, j} \chi_{m ; i, j}$.

Here $w_{i, j}$, etc. are defined by $\int_{-\infty}^{i \Delta t} \chi_{e}\left(\bar{r}, i \Delta t, t^{\prime}\right) \overline{\mathrm{E}}\left(\bar{r}, t^{\prime}\right) d t \approx \Delta t \sum_{j=-\infty}^{i} w_{i, j} \chi_{e, i, j}(\bar{r}) \bar{E}_{j}(\bar{r})$ and so on. It is also found from experiments that the $w_{i, j}$ 's corresponding to the trapezoidal numerical integration, i.e., $w_{i, j}=0 . \S$ for $i=j$, and $w_{i, j}=1$ for $j<i$ are good enough in general.

The equations (2) and (4) are similar to the frequency domain Maxwell equations and the constitution relationship for time-invariant media, respectively. Thus we can write down the solutions to (2) and (4) from the corresponding frequency domain solutions. The rule is simply to substitute $j \omega$ by $\frac{1}{\Delta t}[D]$ and take care of the order of matrix multiplication. From this mathematical duality, the discrete-time plane wave solution in homogeneous time-varying media is obtained directly as

$$
\begin{aligned}
& {[E(x)]=e^{-\frac{x}{c_{0} \Delta t}[y\}}\left[f^{+}\right]+e^{\frac{x}{\varepsilon_{0} \Delta t}[y]}\left[f^{-}\right]} \\
& {[H(x)]=\frac{1}{\eta_{0}}[\eta]^{-1}\left\{e^{-\frac{x}{\varepsilon_{0} \Delta t}[y]}\left[f^{+}\right]-e^{\frac{x}{\varepsilon_{0} \Delta t}[y\}}\left[f^{-}\right]\right\}}
\end{aligned}
$$


Here the plane wave is linearly polarized in the y-direction, $c_{0}=\frac{1}{\sqrt{\varepsilon_{0} \mu_{0}}}, \eta_{0}=\sqrt{\frac{\mu_{0}}{\varepsilon_{0}}}, \quad[\gamma]=\sqrt{\left([D \mathbf{J} \mu]+\frac{c_{0} \Delta t}{\eta_{0}}\left[\sigma_{m}\right)\left([D \mathbf{I}]+\eta_{0} c_{0} \Delta t\left[\sigma_{e}\right]\right.\right.}$, $[\eta]^{-1}=\left(\left[D[\mu]+\frac{c_{0} \Delta t}{\eta_{0}}\left[\sigma_{m}\right]\right)^{-1}[\gamma] ;\right.$ and $\left[f^{+}\right],\left[f^{-}\right]$are constant sequences to be determined by other conditions. Equation (5) can be also derived by manipulating (2) and (4). The part of solution corresponding to $\left[f^{+}\right]$is associated with a wave propagating in the $+x$ direction, while the other part represents a wave propagating in the $-x$ direction. This can be verified by checking their behaviors in free space. Of course, solutions for other kinds of excitations and media structures can be handled in a similar way. Note, however, the rate of change of the media is assumed to be small, such that the reflection due to abrupt change of the media in time can be neglected.

For the special case that the media is time-invariant, including the dispersive media like Debye or Lorentz media, and using causal differentiator such that $d_{i}=0$, for $i<0$, all matrices in (5) become the "staircase matrix," which is the intersection of the Toeplitz matrix and the lower triangular matrix. Nice properties and fast algorithms for computing the matrix functions of staircase matrices in (5) have been given in [4]. Some numerical computations have been done for wave propagations in dispersive and time-varying media. The results will be presented in the conference.

\section{References}

[1] F. R. Morgenthaler, "Velocity modulation of electromagnetic waves," IRE Trans. Microwave Theory Tech., vol. MTT-6, pp. 167-172, April 1958.

[2] W. Ren and B. Q. Gao, "The analysis of $3 \mathrm{~dB}$ microstrip directional coupler in time-varying media by FDTD method," Proc. $2^{\text {nd }}$ International Conference on Microwave and Millimeter Wave Technology, pp. 375-378, 2000.

[3] S. K. Jeng, "Discrete-time electromagnetic theory," 2002 IEEE AP-S International Symposium, San Antonio, TX, June 2002.

[4] I. T. Chiang, Matrix Approaches for Transient Analysis of Complex Transmission Line Circuits Using HWSD and SATD, Ph.D. Thesis, National Taiwan University, Taipei, Taiwan, 2002. 